\title{
lodixanol, a New Isosmotic Nonionic Contrast Agent Compared with lohexol in Cardiac Angiography
}

\author{
James A. Hill, MD, Martin B. Cohen, MD, William H. Kou, MD, G.B. John Mancini, MD, \\ Michael Mansour, MD, Harvey Fountaine, and Jeffrey A. Brinker, MD
}

lodixanol, a now ratio 6 nonionic iodinated contrast agent with an osmolality equal to serum, was compared with iohexol in a randomized, double-blind, parallel study. Two hundred patients undorgoing elective diagnostic cardiac anglography were randomized to iodixanol ( $n=$ 101) or iohexol $(n=99)$. There were $n$ differences noted between the 2 agents in the mean changes in systolic or diastolic blood pressure or heart rate during or immediately after any angiography. However, significantly more patients had a decrease in diastolic blood pressure of $>20 \mathrm{~mm}$ Hg during left coronary angiography with iodixanol. The only significant differences in any electrophysiologic parameter were slightly more PR prolongation during left coronary anglography with iodixanol and more ST-segment depression with iohexol during coronary angiography. Noither was clinically significant. Injection-associated discomfort occumed with both agents, but more patients experienced moderate to severe discomfort with iohexol (52\%) than with iodixanol (17\%) (p<0.001). Only 1 potentially serlous adverse event, ventricular fibrillation with iohexol, was considered related to contrast, and there were no differences noted between the agents. Overall, angiographic quality was equal with all angiograms being assessed as good or excellent in both groups ( $=$ 0.885). In this low-risk population undergoing cardiac anglography, iodixanol is safe and effective without clinically important differences from iohexol. Additional studies in patients at high risk for complications should help further define the role of iodixanol in cardiac angfography.

(Am J Cardiol 1994;74:57-63)

From the University of Florida, Gainesville, Florida; New York Medical College, Westchester County Medical Center, New York, New York; University of Michigan, Ann Arbor, Michigan; University of British Columbia, Vancouver, B.C.; Sterling Winthrop, Collegeville, Pennsylvania; and Johns Hopkins University, Baltimore, Maryland. This study was supported by a grant from Sterling Winthrop, Collegeville, Pennsylvania. Manuscript received September 10, 1993; revised manuscript received November 29, 1993, and accepted November 30 .

Address for reprints: James A. Hill, MD, Division of Cardiology, University of Florida, Box 100277, J. Hillis Miller Health Center, Gainesville, Florida 32610 .
$\mathrm{N}$ onionic iodinated contrast agents have been widely adopted in the cardiac catheterization laboratory; the recent Society for Cardiac Angiography and Interventions registry report indicates that $72 \%$ of diagnostic catheterizations and $77 \%$ of coronary angioplastics are performed using these agents. ${ }^{1}$ There have been numerous studies ${ }^{2-5}$ showing that nonionic low osmolar media produce less hemodynamic and electrophysiologic effects with intracoronary injection, which seems to translate into fewer adverse clinical events. ${ }^{6.7}$ Recently, a newer nonionic agent, iodixanol, has been developed and is formulated to have an osmolality equal to serum. This agent has been shown to be safe in noncardiac applications, ${ }^{8}$ and small studies ${ }^{8,9}$ have suggested that it is also safe for use in cardiac angiography. This study was undertaken to evaluate the hemodynamic and electrophysiologic effects of iodixanol and to compare them with a standard nonionic contrast agent, iohexol.

\section{METHODS}

This study was conducted at 4 sites: the University of Florida in Gainesville, the University of Michigan, New York Medical College Westchester County Medical Center, and the Johns Hopkins Hospital. Patients undergoing elective diagnostic cardiac angiography including both coronary and left ventricular angiography were screened for inclusion. Exclusion criteria were designed to eliminate patients with severe left ventricular dysfunction, aortic stenosis, or unstable clinical syndromes. The study was approved by the respective institutional review boards.

Contrast agents: Iohexol is a nonionic monomeric contrast agent with a molecular weight of 821.14 and iodine content of $46.36 \%$. The concentration used for this study was the standard commercially available formulation (Omnipaque $-350^{\circledR}$ ) containing $350 \mathrm{mg} / \mathrm{ml}$ of iodine with an osmolality of $844 \mathrm{mOsm} / \mathrm{kg}$ of water. Iodixanol (Visipaque $^{\mathrm{TM}}$ ) is a nonionic dimeric contrast agent with a molecular weight of 1550.20 and iodine content of $49.1 \%$. It is formulated to be isosmotic $(290 \mathrm{mOsm} / \mathrm{kg}$ of water) with plasma by the addition of sodium and calcium chloride. The formulation used for this study contained $320 \mathrm{mg} / \mathrm{ml}$ of iodine. The chemical structures of both agents are illustrated in Figure 1.

Catheterization procedure: This was a randomized, double-blind, parallel study. Assignment to receive either iodixanol or iohexol was performed according to a computer-generated randomization scheme specific for each site with catheterization laboratory personnel un- 
aware of patients' clinical data. Angiography was performed using standard methods, and specifics of preparation and technique were left to the discretion of the investigator. The only exceptions were that use of atropine as prophylaxis for bradyarrhythmias was not allowed and that a femoral arterial sheath with a sideport $1 \mathrm{Fr}$ size larger than the catheter was used.

Measurements: Systemic blood pressure was monitored from the sideport of the femoral sheath. During coronary injections, systolic and diastolic blood pressure, heart rate, PR and QT intervals (milliseconds), and ST-segment and T-wave amplitude (millivolts) were measured. Immediately after left ventricular injection, left ventricular systolic and end-diastolic pressure were measured. During the first injection of any type (i.e., right coronary, left coronary, or left ventricular) all parameters were recorded immediately before, during, and continuously for the first 15 seconds, and again at 30,60 , and 120 seconds or until the parameter returned to baseline. With subsequent injections, these parameters were followed for 60 seconds or until the parameter returned to baseline. Any arrhythmias present after injection were also recorded, and QTc was calculated using a standard formula. After the procedure, blood pressure and heart rate were measured at 30 and 60 minutes and according to local protocol thereafter. Contrast volume with each injection and total contrast volume were recorded. Blood samples were collected at baseline, 1 hour, and 1 day after the last contrast injection and analyzed for complete blood count and chemistries. Serum creatinine and urea nitrogen were also measured 2 and 3 days after angiography.

Patients were followed for adverse events during and for 72 hours after the procedure. The actions required, intensity of the events, and outcome were tabulated. Serious adverse events included any experience that was fatal, life-threatening, or permanently disabling, or required further hospitalization. Judgment was made by the investigator regarding the causality of the event and its relation to the contrast material. Subjects were asked to evaluate any discomfort during injections according to type (heat, coldness, etc.), location, duration, and intensity.

Each injection was evaluated for the quality of visualization using cineangiograms by the blinded individual investigators. An angiogram was considered nondiagnostic if there was insufficient contrast enhancement to make a diagnosis. Angingrams considered diagnostic had sufficient contrast enhancement and were further divided into good and excellent based on qualitative observer estimation.

Statistical analysis: Data from all sites were combined for analysis. Fisher's exact test (2-tailed) was used for comparing contrast agent groups with respect to overall quality of visualization, adverse events, injection-associated discomfort, arrhythmias, and serum creatinine (classification being above the reference range, or change from baseline $>40 \%$ of the span of the reference range). Hemodynamic parameters were analyzed by using the maximal absolute change from baseline to classify each patient as having an increase, decrease, or no change. Fisher's exact test was then used to compute group differences. Electrophysiologic parameters were analyzed using repeated-measures analysis of variance based on ranks. Differences among centers were quantified using analysis of variance with contrast agent, and center and contrast by center interaction terms in the model.

\section{RESULTS}

Patient demographics: Two hundred patients were randomized: 101 to iodixanol (81 men) and 99 to iohexol ( 87 men). The mean age in the iodixanol group was 61

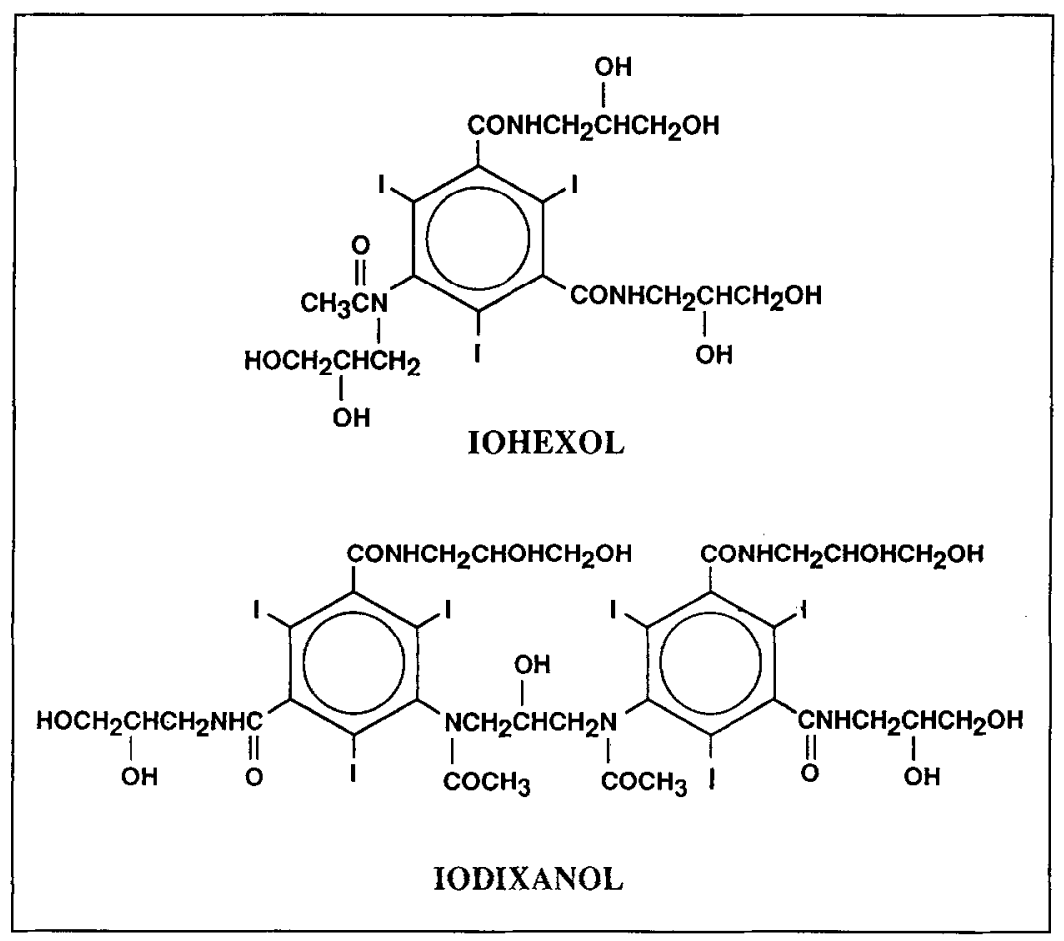

FGURE 1. Chemical structures of iohexol and iodixanol. 
\pm 10 years and in the iohexol group $59 \pm 11$ years $(\mathrm{p}=$ 0.291 ). All patients completed the study. There were no differences between the 2 groups with regard to indication for catheterization. Suspicion of or definite coronary artery disease was the indication for study in $92 \%$ of patients. Coronary artery disease was found in $84 \%$ and $80 \%$ of patients with iodixanol and iohexol, respectively.

Procedural variables: Procedure duration $(39 \pm 14$ vs $40 \pm 18$ minutes), contrast volume ( $103 \pm 46 \mathrm{ml}$ vs $103 \pm 37 \mathrm{ml}$ ), and average number of injections $(9 \pm 2$ vs $9 \pm 3$ ) were not different between the iodixanol and iohexol groups, respectively. In both groups, $97 \%$ of pa- tients underwent left ventriculography, and all patients except 1 in the iohexol group underwent coronary angiography.

Image quality: No patient in either group had a nondiagnostic study. Overall, in the iodixanol group, the quality of the angiograms was assessed as good in $41 \%$ and excellent in $59 \%$. In the iohexol group the angiograms were assessed as good in $39 \%$ and excellent in $61 \%$ (iodixanol vs iohexol, $\mathrm{p}=0.885$ ).

Hemodynamic effects: Mean changes in systolic and diastolic pressure and heart rate occurring with first right and left coronary injection are shown in Figures 2

\section{RIGHT CORONARY INJECTION}

SYSTOLIC PRESSURE

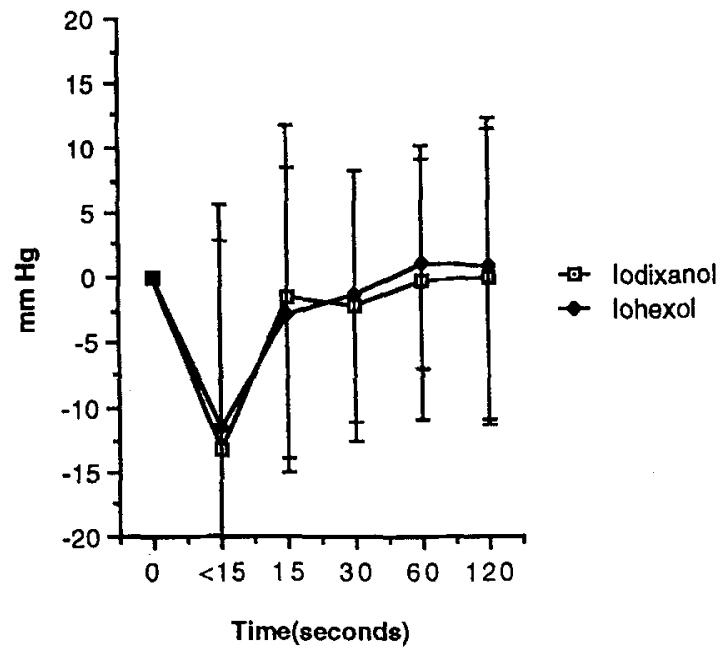

DIASTOLIC PRESSURE

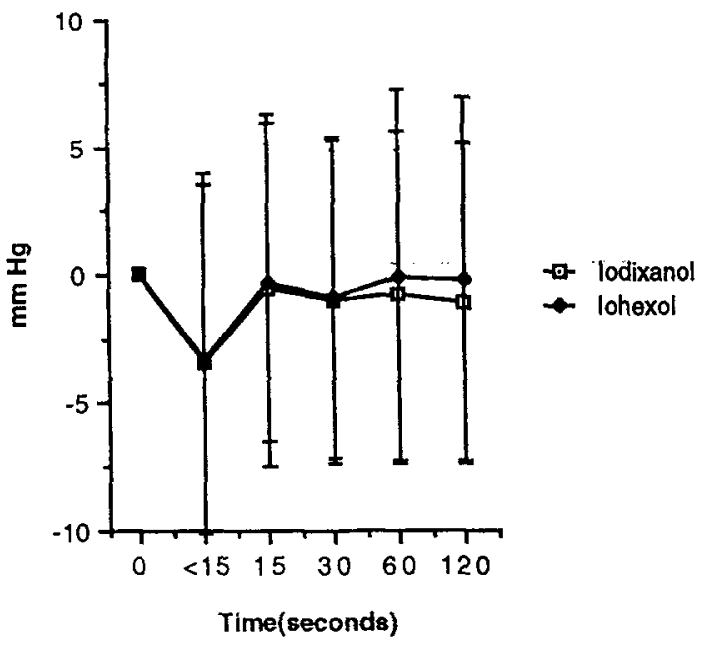

HEART RATE

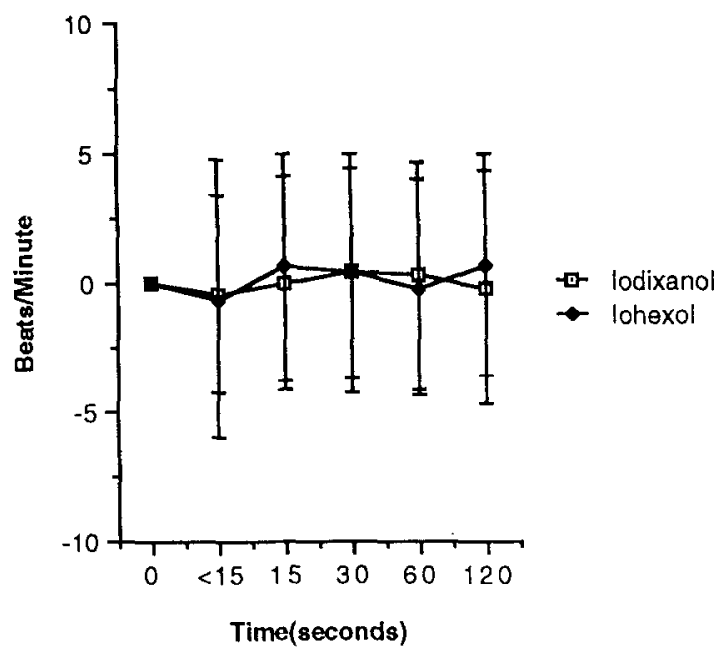

FGURE 2. Hemodynamic changes after right coronary arterlography. No significant differences are noted between iohexol and iodlxanol. 


\begin{tabular}{|c|c|c|c|c|c|}
\hline & \multicolumn{2}{|c|}{ lodixanol } & \multicolumn{2}{|c|}{ lohexol } & \multirow[b]{2}{*}{$p$ Value } \\
\hline & Increase & Decrease & Increase & Decrease & \\
\hline \multicolumn{6}{|l|}{ Right coronary } \\
\hline Systolic BP > $20 \mathrm{~mm} \mathrm{Hg}$ & 10 & 42 & 7 & 37 & 0.511 \\
\hline Diastolic BP >20 mm Hg & 4 & 5 & 4 & 3 & 0.922 \\
\hline Heart rate $>15$ beats $/ \mathrm{min}$ & 3 & 1 & 3 & 3 & 0.620 \\
\hline \multicolumn{6}{|l|}{ Left coronary } \\
\hline Systolic BP > $20 \mathrm{~mm} \mathrm{Hg}$ & 4 & 62 & 11 & 49 & 0.070 \\
\hline Diastolic BP >20 mm Hg & 3 & 13 & 2 & 3 & 0.025 \\
\hline Heart rate $>15$ beats $/ \mathrm{min}$ & 2 & 9 & 4 & 2 & 0.082 \\
\hline \multicolumn{6}{|l|}{ Left ventricle } \\
\hline Systolic BP > $20 \mathrm{~mm} \mathrm{Hg}$ & 5 & 20 & 11 & 15 & 0.298 \\
\hline Diastolic BP $>4 \mathrm{~mm} \mathrm{Hg}$ & 41 & 21 & 56 & 17 & 0.102 \\
\hline Heart rate $>15$ beats $/ \mathrm{min}$ & 5 & 0 & 4 & 1 & 0.872 \\
\hline
\end{tabular}

\begin{tabular}{|c|c|c|c|c|c|}
\hline \multirow[b]{2}{*}{ Time After Injection } & \multirow[b]{2}{*}{ Baseline (ms) } & \multicolumn{4}{|c|}{ Seconds } \\
\hline & & $15^{*}$ & $30 t$ & 60 & 120 \\
\hline \multicolumn{6}{|l|}{$\begin{array}{l}\text { Left coronary } \\
\text { PR interval }\end{array}$} \\
\hline lodixanol & $183 \pm 30$ & $1 \pm 6$ & $1 \pm 7$ & $0 \pm 7$ & $2 \pm 7$ \\
\hline lohexol & $192 \pm 38$ & $-1 \pm 7$ & $-1 \pm 7$ & $0 \pm 6$ & $1 \pm 8$ \\
\hline \multicolumn{6}{|l|}{ QT interval } \\
\hline lodixanol & $397 \pm 40$ & $1 \pm 5$ & $0 \pm 6$ & $0 \pm 6$ & $0 \pm 6$ \\
\hline lohexol & $393 \pm 41$ & $2 \pm 5$ & $1 \pm 6$ & $1 \pm 5$ & $1 \pm 5$ \\
\hline \multicolumn{6}{|l|}{ QTc interval } \\
\hline lodixanol & $419 \pm 43$ & $1 \pm 6$ & $0 \pm 7$ & $0 \pm 7$ & $0 \pm 7$ \\
\hline lohexol & $422 \pm 39$ & $2 \pm 6$ & $1 \pm 7$ & $1 \pm 6$ & $1 \pm 6$ \\
\hline \multicolumn{6}{|l|}{ Right coronary } \\
\hline \multicolumn{6}{|l|}{$\mathrm{PR}$ interval } \\
\hline lodixanol & $185 \pm 30$ & $0.4 \pm 7$ & $1 \pm 8$ & $1 \pm 9$ & $0 \pm 7$ \\
\hline lohexol & $190 \pm 30$ & $1 \pm 8$ & $2 \pm 7$ & $2 \pm 6$ & $3 \pm 7$ \\
\hline \multicolumn{6}{|l|}{ QT interval } \\
\hline lodixanol & $400 \pm 42$ & $1 \pm 7$ & $0 \pm 7$ & $-1 \pm 6$ & $0 \pm 5$ \\
\hline lohexol & $394 \pm 41$ & $1 \pm 5$ & $0 \pm 6$ & $0 \pm 5$ & $1 \pm 7$ \\
\hline \multicolumn{6}{|l|}{ QTc interval } \\
\hline lodixanol & $421 \pm 42$ & $1 \pm 7$ & $1 \pm 8$ & $-1 \pm 7$ & $0 \pm 6$ \\
\hline lohexol & $424 \pm 36$ & $1 \pm 6$ & $0 \pm 6$ & $0 \pm 5$ & $1 \pm 7$ \\
\hline
\end{tabular}

and 3. Mean changes in systolic and end-diastolic pressures and heart rate occurring with left ventriculography are shown in Figure 4.

Because of the variability in changes in blood pressure during injection into both coronaries and the left ventricle, large changes were evaluated. The number of patients with a $>20 \mathrm{~mm} \mathrm{Hg}$ change in systolic and diastolic pressure during coronary angiography and $>4 \mathrm{~mm}$ $\mathrm{Hg}$ change in end-diastolic pressure after ventricular injection are listed in Table I. Significantly more patients had a decrease in diastolic blood pressure of $>20 \mathrm{~mm}$ $\mathrm{Hg}$ during left coronary angiography with iodixanol. There were no differences noted between the 2 agents during right coronary angiography or ventriculography.

Electrophysiologic offocts: Changes in the PR, QT, and QTc intervals were noted during left ventricular injection, but there was wide variability among patients with changes from baseline ranging from $-38 \%$ to $37 \%$. However, no significant differences were noted between the agents. Electrophysiologic changes occurring immediately after coronary injection are listed in Table II. There was a significant difference in the PR interval during left but not right coronary artery injection between iodixanol, which caused maximal prolongation of $<1.5 \%$, and iohexol during the time of monitoring. There was significantly more ST-segment depression in the iohexol than in the iodixanol group with both left and right coronary angiography, but this was not found to be clinically relevant $(p<0.023)$.

Many patients, $74 \%$ with iodixanol and $77 \%$ with iohexol, had some sort of arrhythmia during catheterization ( $\mathrm{p}=\mathrm{NS}$ ), both before and after contrast administration. There was 1 episode of nonsustained ventricular tachycardia not requiring treatment and 1 episode of ventricular fibrillation, both occurring with iohexol.

Advorse ovents: There were no significant differences in adverse events when the 2 agents were compared, with $35 \%$ of patients in both groups experienc- 
ing a generally mild adverse event. Only 5 ( 2 with iodixanol and 3 with iohexol) were considered to be definitely related to the contrast material. In all, there were only 4 serious events in 3 patients (3\%) with iodixanol and 5 events in 5 patients $(5 \%)$ with iohexol. Only 1 serious event, ventricular fibrillation with iohexol, was considered definitely related to contrast. Two others, pulmonary edema and severe chest pain, both with iohexol, were considered of uncertain relation to contrast. None of the 4 serious events occurring with iodixanol were considered to be related to contrast. There were no deaths and all patients recovered without sequelae.
After the procedure, there were variations in heart rate and blood pressure that were considered to be of clinical importance in $4 \%$ of the iodixanol group and $13 \%$ of the iohexol group $(p=0.024)$, but none were considered to be directiy attributable to contrast. Analysis of laboratory data showed no significant trends that suggested toxicity from either agent. Specifically, 6 patients with iodixanol and 7 with iohexol had increases in serum creatinine, both $>40 \%$ of the span of the reference range and above the normal range. Only $2 \mathrm{pa}-$ tients had increases in serum creatinine of $>0.5 \mathrm{mg} / \mathrm{dl} 3$ days after catheterization. These patients received io-

\section{LEFT CORONARY INJECTION}

SYSTOLIC PRESSURE

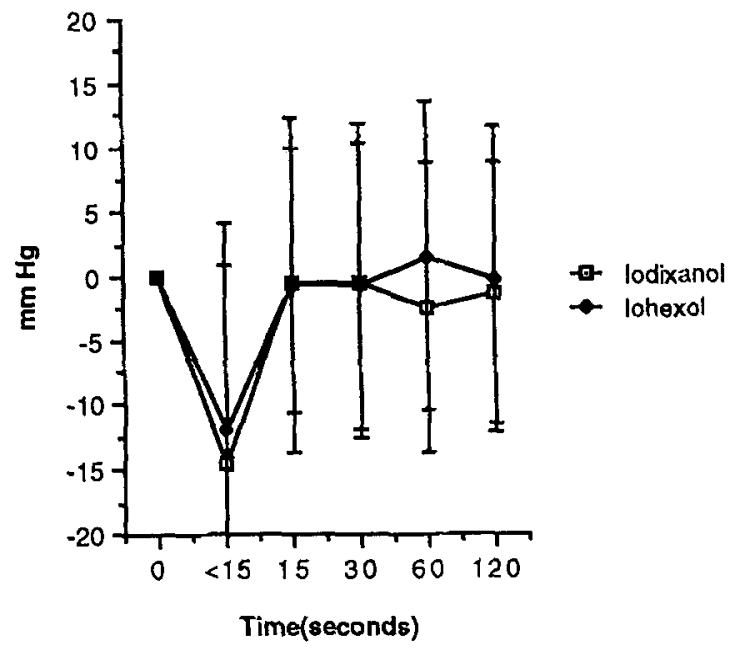

DIASTOLIC PRESSURE

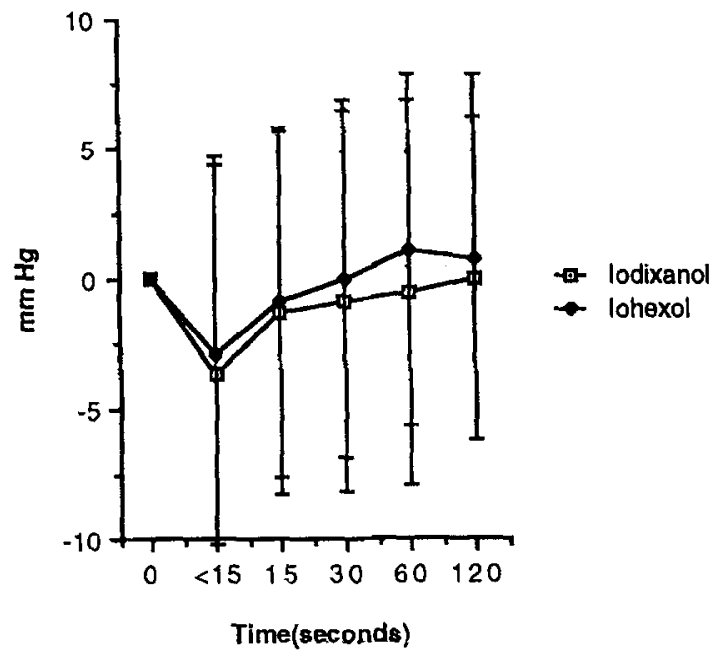

MEART RATE

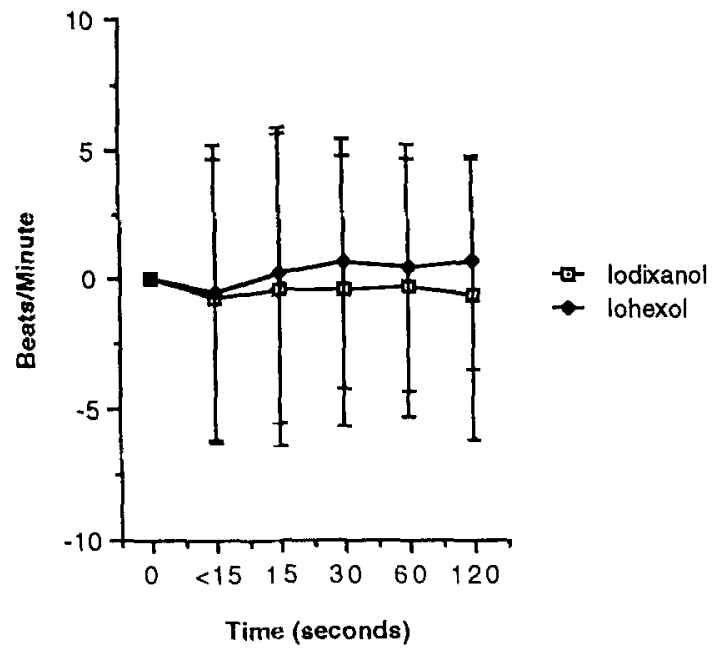

FiQuRE 3. Hemodynamic changes efter left coronary arteriography. No significant differences are noted between lohexol and lodixanol. 
hexol and had baseline creatinine levels of 1.3 and 1.9 $\mathrm{mg} / \mathrm{dl}$, and maximal serum creatinine levels of 3.7 and $2.6 \mathrm{mg} / \mathrm{dl}$, respectively.

Injection-associated discomfort: Injection-associated discomfort was reported in $77 \%$ and $86 \%$ of patients receiving iodixanol and iohexol, respectively $(\mathrm{p}=$ 0.145 ). As would be expected, in the majority, this was a transient warm feeling, which lasted $<2$ minutes. However, more patients experienced moderate to severe discomfort with iohexol (52\%) than with iodixanol (17\%) (p <0.001).
Because of differences in individual practices among centers, there were some minor intercenter differences found in patient demographics and other parameters that were consistent between the agents.

\section{DIScussion}

While additives and calcium-binding properties play a role in many of the hemodynamic and electrophysiologic effects caused by contrast agents, high osmolality also has a significant effect on a variety of physiologic processes. Lower osmolality is achieved either by in-

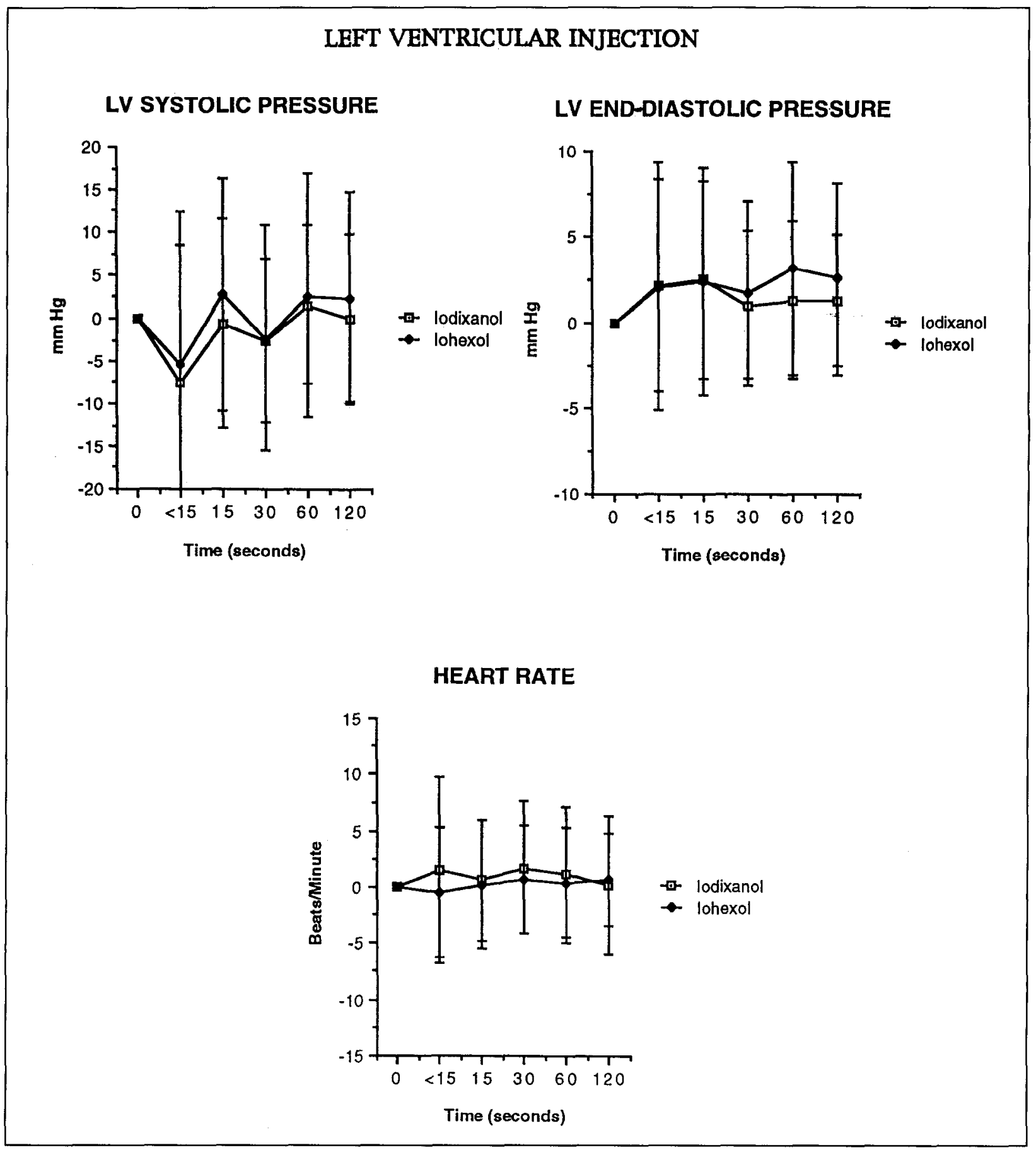

FGURE 4. Hemodynamic changes after left ventrlcular (LV) anglography. No significant differences are noted between iohexol and lodixanol. 
creasing the number of iodine atoms per molecule of the contrast agent, as with the ionic dimeric agent ioxaglate, or by eliminating the need to ionize in solution, as with the conventional nonionic agents iopamidol, ioversol, or iohexol. In either case, these agents provide 3 iodine atoms per osmotically active particle in solution, thus making them ratio 3 agents with approximately half of the osmolality of ratio 1.5 agents. Iodixanol contains 6 iodine atoms per particle and does not ionize in solution, thus making it a ratio 6 agent. With the addition of sodium and calcium the osmolality is equal to serum.

In vitro experiments suggest that iodixanol is not significantly different from other nonionic agents as to changes in red cell morphology and aggregation induced by contact. ${ }^{10,11}$ Data from intact animal and other models suggest favorable hemodynamic effects of iodixanol when compared with conventional nonionic agents. ${ }^{12-14}$

Published clinical experience with iodixanol is limited. Gavant and Siegle ${ }^{15}$ reported it to be safe and effective for intravenous use for excretory urography. Summarized initial data from phase I and II trials in Europe using iodixanol for intraarterial and intravenous use also show a good safety profile. ${ }^{8}$ Klow et al $^{9}$ performed cardiac angiography with iodixanol in 14 stable patients, and then compared it with iohexol in 72 other patients and found good patient tolerance. They found no heart rate or hemodynamic changes during left ventricular angiography but did not discuss these parameters after coronary injection.

Nonionic contrast agents in general have been previously shown to have much less severe hemodynamic and electrophysiologic effects than high osmolar agents when used for cardiac angiography. It is not clear whether this is solely due to the decrease in osmolality or whether there is less chemotoxicity with these agents. Iodixanol has a lower osmolality than available nonionic agents, but has sodium and calcium added to the formulation. Whether the addition of these electrolytes in a ratio similar to blood interacts with the reduction in osmolality in some way to alter the toxicity is not known. It would appear from the current study that further improvement in clinically relevant effects of osmolality may not be measurable when compared with the improvement achieved with conventional nonionic con- trast, at least in low-risk patients. Studies in patients at high risk for complications are necessary to help further define the role of iodixanol in cardiac angiography.

1. Johnson LW, Krone R. Cardiac catheterization 1991: a report of the registry of the Suciely for Cardiac Angiography and Interventions (SCA\&I). Cathet Cardiovasc Diagn 1993;28:219-220.

2. Gertz EW, Wisneski JA, Chiu D, Akin JR, Hu C. Clinical superiority of a new nonionic contrast agent (iopamidol) for cardiac angiography. $J$ Am Coll Cardiol 1985;5:250-258.

3. Mancini GBJ, Bloomquist JN, Bhargava V, Stein JB, Lew W, Slutsky RA, Shabetai $R$, Higgins $C B$. Hemodynamic and electrocardiographic effects in man of a new nonionic contrast agent (iohexol); advantages over standard ionic agents. Am $J$ Cardiol 1983;51:1218-1222.

4. Murdock CJ, Davis MJE, Ireland MA, Gibbons FA, Cope GD. Comparison of meglumine sodium diatrizoate, iopamidol, and iohexol for coronary angiography and ventriculography. Cathet Cardiovasc Diagn 1990;19:179-183.

5. Ciuffo AA, Fuchs RM, Guzman PA, Brin KP, Kross DE, Weiss JL, Feroli RW Brinker JA. Benefits of nonionic contrast in coronary angiography: preliminary results of a randomized double-blind trial comparing iopamidol with Renografin-76. Invest Radiol 1984;19:\$197-S202.

6. Hill $\mathrm{J} \Lambda$, Winniford $M$, Cohen MB, Van Fossen DB, Murphy MJ, Halpcrn E, Ludbrook PA, Wexler L, Rudnick MR, Goldfarb S. Multicenter trial of ionic vs nonionic contrast media for cardiac angiography. Am J Cardiol 1993;72:770-775. 7. Steinberg EP, Moore RD, Powe NR, Gopalan R, Davidoff AI, I itt M, Graziann S, Brinker JA. Safety and cost effectiveness of high-osmolality as compared with low-osmolality contrast material in patients undergoing cardiac catheterization. $N$ Engl I Med 1992;326:425-430.

8. Bolstad B, Borch KW, Grynne BH, Lundby B, Nossen JOE, Kloster YF, Kristoffersen DT, Andrew E. Safety and tolerability of iodixanol: a dimeric, nonionic contrast medium: an emphasis on European clinical phases I and II. Invest Radiol 1991;26:\$20!-\$204.

9. Klow NE, Levorstad K, Berg KJ, Brodahl U, Endersen K, Kristoffersen DT, Laake B, Simonsen S, Tofte AJ, Lundby B. Iodixanol in cardioangiography in patients with coronary artery disease: tolerability, cardiac and renal effects. Acta Rudiol 1993;34:72-77

10. Aspelin P, Nilsson PE, Schmid-Schonbein H, Schroder S, Simon R. Effect of four non-ionic contrast media on red blood cells in vitro: 1. Morphology. Acta Radiol 1987(suppl);370:79-83.

11. Aspelin $P$, Nilsson PE, Schmid-Schonbein $H$, Schroder $S$, Simon R. Effect of four non-ionic contrast media on red blood cells in vitro: 2 . Aggregation. Acta Radiol 1987(suppl);370:85-91.

12. Almen T. Effects of iodixanol, iopentol, iohexol and metrizoate on femoral blood flow after injection into the femoral artey of the dog. Acta Radiol 1987 (suppl);370:69-72.

13. Dundore RL, Silver PJ, Exrin AM, Lee KC, Buchholz RA, Van Aller G, Clas DM, Roth GM, Harnish PP, Bailey DM, Pagani ED. The effects of iodixanol and iopanidol on hemodynamic and cardiac electrophysiologic parameters in vitro and in vivo. Invest Radiol 1991;26:715-721.

14. Klow NE, Mortensen E, Refsum HJ. Left ventricular systolic and diastolic function during coronary angiography before and after acute left ventricular failure in dogs: a comparison between iodixanol, iohexol and ioxaglate. Acta Radiol 1991;32: 124-129.

15. Gavant ML, Siegle RL. Iodixanol in excretory urography: initial clinical experience with a nonionic, dimeric (ratio 6:1) contrast medium. Radiology 1992;183: $515-518$. 